\title{
Airway stents
}

\section{Erik Folch, Colleen Keyes}

Division of Pulmonary and Critical Care Medicine, Section of Interventional Pulmonary, Massachusetts General Hospital, Harvard Medical School, Boston, MA, USA

Correspondence to: Erik Folch. Division of Pulmonary and Critical Care Medicine, Section of Interventional Pulmonary, Massachusetts General Hospital, Harvard Medical School, 55 Fruit Street, Boston, MA 02114, USA. Email: efolch@mgh.harvard.edu.

\begin{abstract}
Stents and tubes to maintain the patency of the airways are commonly used for malignant obstruction and are occasionally employed in benign disease. Malignant airway obstruction usually results from direct involvement of bronchogenic carcinoma, or by extension of carcinomas occurring in the esophagus or the thyroid. External compression from lymph nodes or metastatic disease from other organs can also cause central airway obstruction. Most malignant airway lesions are surgically inoperable due to advanced disease stage and require multimodality palliation, including stent placement. As with any other medical device, stents have significantly evolved over the last 50 years and deserve an in-depth understanding of their true capabilities and complications. Not every silicone stent is created equal and the same holds for metallic stents. Herein, we present an overview of the topic as well as some of the more practical and controversial issues surrounding airway stents. We also try to dispel the myths surrounding stent removal and their supposed use only in central airways. At the end, we come to the long-held conclusion that stents should not be used as first line treatment of choice, but after ruling out the possibility of curative surgical resection or repair.
\end{abstract}

Keywords: Airway stents; airway obstruction/therapy; tracheal neoplasms/therapy; prosthesis design; prosthesis implantation; bronchi/pathology

Submitted Jan 19, 2018. Accepted for publication Feb 27, 2018.

doi: $10.21037 /$ acs.2018.03.08

View this article at: http://dx.doi.org/10.21037/acs.2018.03.08

\section{Introduction}

An airway stent is an endobronchial prosthesis of various materials that supports and maintains patency of the hollow tubular airway structure (1). An airway stent may impede extension of a tumor into the airway, may help in the healing or management of airway fistulas, or may support the airway wall against collapse or external compression.

\section{Indications and contraindications for airway stents}

Airway stenting is indicated in a variety of malignant and benign processes (Table 1). Independent of the indication, prior to any stent placement, the thoracic surgeon or interventional pulmonologist must be sure that the patient does not have any surgically curable airway disease. Stenting may complicate or preclude surgery in some cases, particularly when it extends the lesion to $>4 \mathrm{~cm}$ in length, a commonly used limit for tracheal resection. Furthermore, placement of an airway stent can create local inflammation and mucosal injury that may interfere with anastomotic healing following airway resection and reconstruction (2). The most common indications for stenting include minimizing extrinsic compression from tumors or lymphadenopathy, maintaining patency after bronchoscopic tumor removal due to intrinsic obstruction, healing airway fistulas and anastomotic dehiscence post lung transplantation and addressing very selected cases of benign airway disease $(3,4)$. It is important to note that in general, metallic stents are not considered first-line of therapy in patients with benign airway obstruction and should only be used if everything else fails (5).

The use of airway stents frequently elicits visceral reaction from some thoracic surgeons and interventional pulmonologists. These reflexive responses are the result of 


Table 1 Indications for airway stenting
Indication: malignant disease
Malignant airway obstruction from extrinsic compression
Endobronchial tumor with residual obstruction after
multimodality thermal therapy
Mixed endobronchial and extrinsic tumor
Loss of cartilage support from tumor destruction
Malignant tracheoesophageal fistula
Indication: benign disease
Complex benign stricture or stenosis $>4$ cm in length
Benign stricture or stenosis in inoperable patient
Post-transplant airway stenosis
Tracheobronchomalacia: therapeutic trial before tracheoplasty
Benign tracheoesophageal fistula

catastrophic cases in the past, or deeply ingrained in their psyche by their mentors. When this occurs, it is important to objectively analyze the specific case, the available options for that particular patient and the solid evidence behind the biomaterials and engineering of current airway stents. In other words, be evidence-based and not dogmatic. There are only a few absolute contraindications to stent placement. In general, patients medically able to undergo surgical interventions to cure benign or malignant conditions should not have an artificial airway prosthesis placed (4). Also, very severe impairment in functional capacity and very limited survival expectancy should preclude stenting. However, recent advances in chemotherapy and radiation for lung cancer challenge our prior assumption (6).

If there is no viable lung tissue beyond the stenosis, stenting should also not be pursued (1). In most cases, careful review of the computed tomography and balloon dilation of the stenosis will reveal if distal airways are patent and thus worthy of reestablishing patency. Relative contraindications include individuals unable to tolerate general anesthesia or moderate sedation (7). In terms of preparation, the clinician should exercise self-restraint and consider if this is the right setting for placement of an airway prosthesis. The most experienced person who can place such a device should be enlisted and a plan should be constructed for contingencies such as airway obstruction during placement, bleeding, or misplacement from the desired location.

\section{Types of airway stents and materials}

The "ideal" stent has not yet been developed. In theory, such an ideal stent should be stable, strong enough to withhold the external compressive forces that compromise the lumen patency, biocompatible (i.e., non-irritating), available in all necessary sizes, resistant to migration, easily deployed and removed and can act as a barrier to inward growth of tumor while being flexible enough to conform to the different luminal irregularities. However, the ideal stent does not currently exist. At the time of writing of this manuscript, the available airway stent materials include silicone, nitinol, stainless steel and hybrid stents. Recent advances in 3-D printing may revolutionize stenting, but medical-grade materials are in the development stage (8).

\section{Is the material the most important factor? Silicone $v s$. metallic vs. hybrid stents}

The material of the stent is not the most important factor to consider when choosing an adequate stent. The selection should be made based on the specific disease, anatomy and clinical situation. Likewise, the experience of the clinician with a specific stent should not be the key factor deciding which stent to place. A rapid referral to a colleague is better than a lengthy and complicated course. The ease of placement of metallic stents (i.e., without the absolute need for rigid bronchoscopy) has popularized their use in malignant and benign disease (9). However, there has been a significant shift towards the use of silicone stents in benign diseases. In 2005, the US Food and Drug Administration (FDA) issued a warning stating that the use of metallic stents should be avoided in benign diseases (5). This warning followed the reports of significant complications in benign airway diseases, when stents were inevitably susceptible based on a relatively longer patient life expectancy and, therefore, longer endobronchial occupancy periods compared to malignant disease indications. It is also important to remember that at the time of that FDA recommendation, the available metallic stents had different biomechanical properties, were mostly uncovered, and, in some cases, repurposed from use in other organs such as the colon, esophagus or biliary tree. Nevertheless, the recommendation stands true today, and should be explicitly considered as follows:

* Use metallic tracheal stents in patients with benign airway disorders only after thoroughly exploring all 
other treatment options (such as tracheal surgical procedures or placement of silicone stents). Using metallic tracheal stents as a bridge to other therapies is not recommended, because removal of the metallic stent can result in serious complications;

* If a metallic tracheal stent is the only option for a patient, insertion should be done by a physician trained or experienced in metallic tracheal stent procedures;

* If removal is necessary, the procedure should be performed by a physician trained or experienced in removing metallic tracheal stents;

* Always review the labelling before using the device, especially the indications for use, warnings and precautions. Select patients carefully.

Table 2 shows the characteristics of the individual stents.

\section{Silicone stents}

Silicone is a synthetic material created of silicone elastomers or polydimethylsiloxane. Silicone has firmness, stability at high temperatures and elicits minimal tissue reactivity (10). It is easily molded and can give various degrees of firmness and flexibility. Reinforcement of the silicone with polypropylene, polyamide and carbon fiber fibers can confer greater mechanical strength and resistance to the silicone matrix (11). Silicone stents are comparatively less expensive, well tolerated, and have the sufficient firmness to resist extrinsic compression. In addition, they can be easily modified by cutting a portion of the stent, allowing customization to the airway anatomy prior to deployment (Figure 1). In general, rigid bronchoscopy is needed for deployment as well as general anesthesia; however, insertion using the flexible bronchoscope has been described (12). Once deployed, they can be repositioned or removed using the rigid grasping forceps.

Silicone stents come in various lengths, shapes, diameters and durometers (hardness of polymers and elastomers). Custom prostheses can be ordered from several manufacturers (Table 2). "Y"-shaped stents are useful in cases of bilateral bronchial involvement, to maintain patency at the main carina, or to prevent migration of a distal silicone tracheal stent. The significant advantages of silicone stents are the ability to be easily repositioned and removed as well as the possibility of customization by making orifices in the desired location. However, tubular silicone stents have a higher risk of migration, and repeated bronchoscopic procedures may be needed for repositioning (Figure 2A) $(9,13,14)$. Obstruction from accumulated secretions may occur as well as from granulation tissue growth at the proximal or distal ends (Figure 2B,C). In addition, ignition during other endobronchial treatments (i.e., laser therapy) is possible. The three most common silicone stents are the Montgomery T-tube, the tubular stent and the Y-stent. Complications include migration with a rate of $9.5 \%$ and obstruction due to secretions with a rate of $3.6 \%(15,16)$.

\section{Metallic and hybrid stents}

Metallic stents may be subdivided into self-expandable metallic stents (SEMS) and fixed-diameter stents that require balloon dilatation. Metal stents are available in covered and uncovered varieties. The initial uncovered metallic stents (i.e., Gianturco, Wallstent, Palmaz) were repurposed models designed for use in the gastrointestinal and the vascular fields, and probably accounted for the early reports of serious complications (17). Most of these initial metallic stents (i.e., Gianturco, Palmaz, etc.) with their sharp ends are now rarely used. Metallic stents have many attractive advantages. They can be inserted with topical anesthesia and moderate sedation via flexible bronchoscopy, When compared to silicone stents, metallic stents have; a better internal-to-external diameter ratio, resulting in a larger airway lumen; are radio-opaque, making them easy to spot on radiographic films; and have a lower incidence of migration $(18,19)$. Uncovered stents have the additional benefits of not creating obstruction across bronchial lobar orifices and, in theory, do not interrupt the mucociliary clearance (13).

On the other hand, uncovered metallic stents can be difficult to remove, and tumor or granulation tissue may grow through the mesh/stent orifices (Figure 3). Serious complications have been reported, including airway or vascular perforation (20). Also concerning is the fact that complications of metallic stents tend to occur more frequently in benign diseases, for reasons previously described. Currently, the role of uncovered metallic stents is limited, however, there are specific indications for their use, such as anastomotic dehiscence following lung transplantation (21-23).

The new generation stents are made from alloys. The most popular composition is nitinol (titanium and nickel alloys); a super-elastic biomaterial that may undergo large deformations in size and shape. Nitinol-made stents have "shape memory" that decreases risk of airway perforation, because they do not change in length once expanded, and are flexible enough to change in shape with cough 


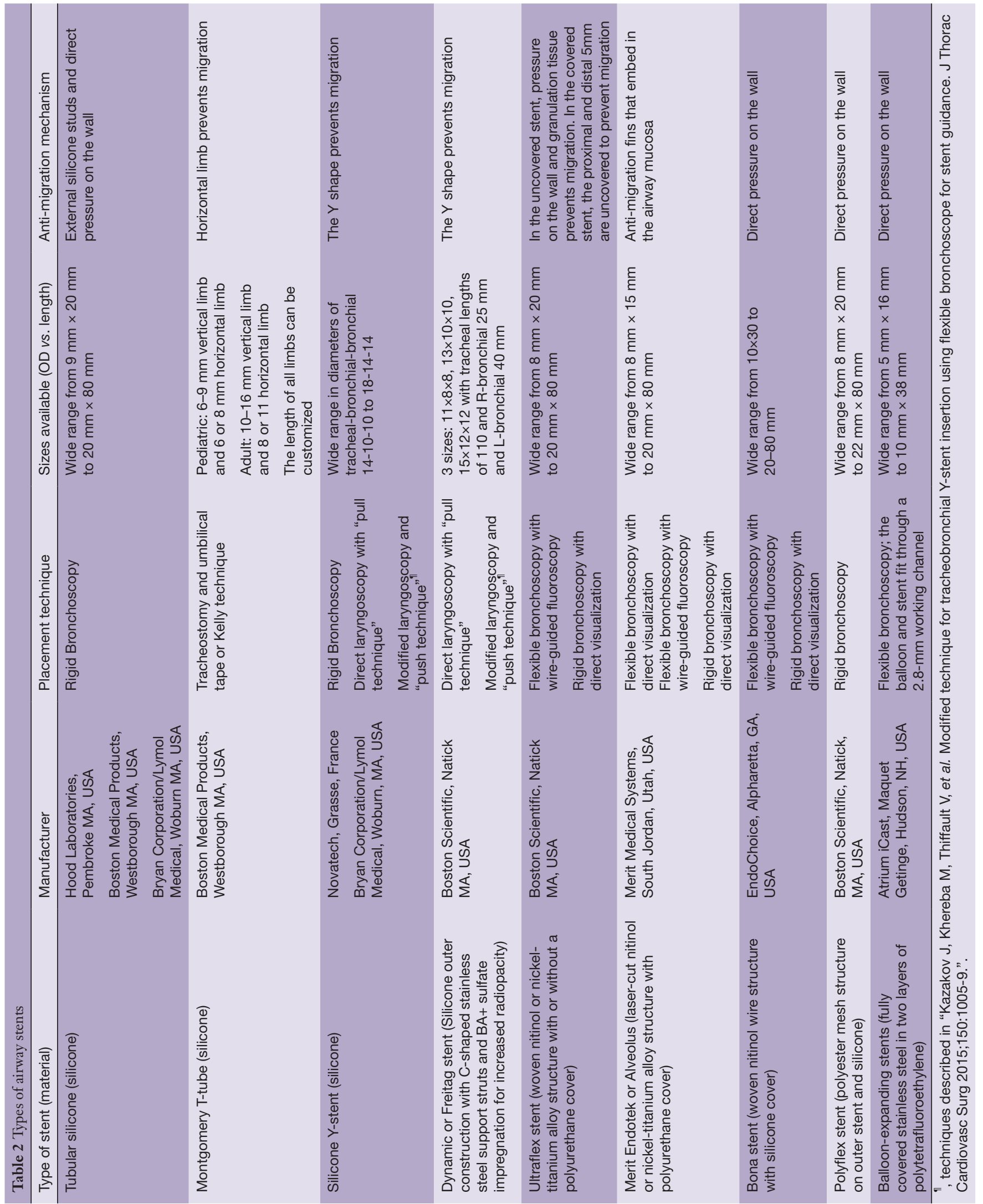




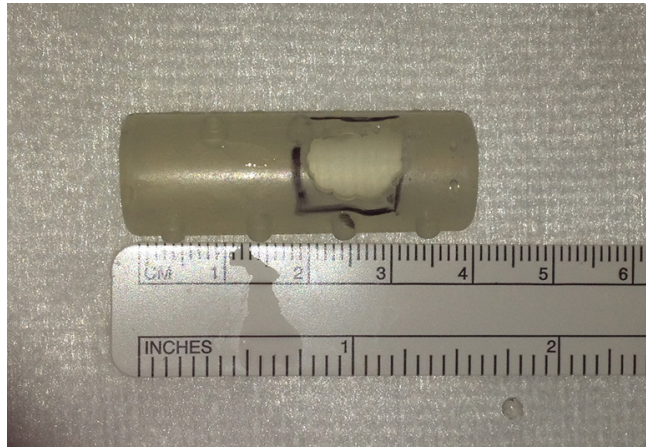

Figure 1 Customized silicone stent.
$(1,24)$. With the idea of incorporating the benefits and minimizing the drawbacks of silicone and bare metal stents, the "hybrid stents," or covered metallic stents, were created. These stents incorporate the polymer and metal alloy technology previously elucidated. Covered metallic stents have the advantage of minimal tissue ingrowth and easier manipulation. Polyurethane, polytetrafluoroethylene (PTFE) and silicone have been used as the covering material for hybrid stents. Degradation of the polyurethane membrane by respiratory secretions has been described (25). PTFE is more chemical resistant; however, a $13 \%$ rate of PTFE separation from the stent at the site of polyurethane
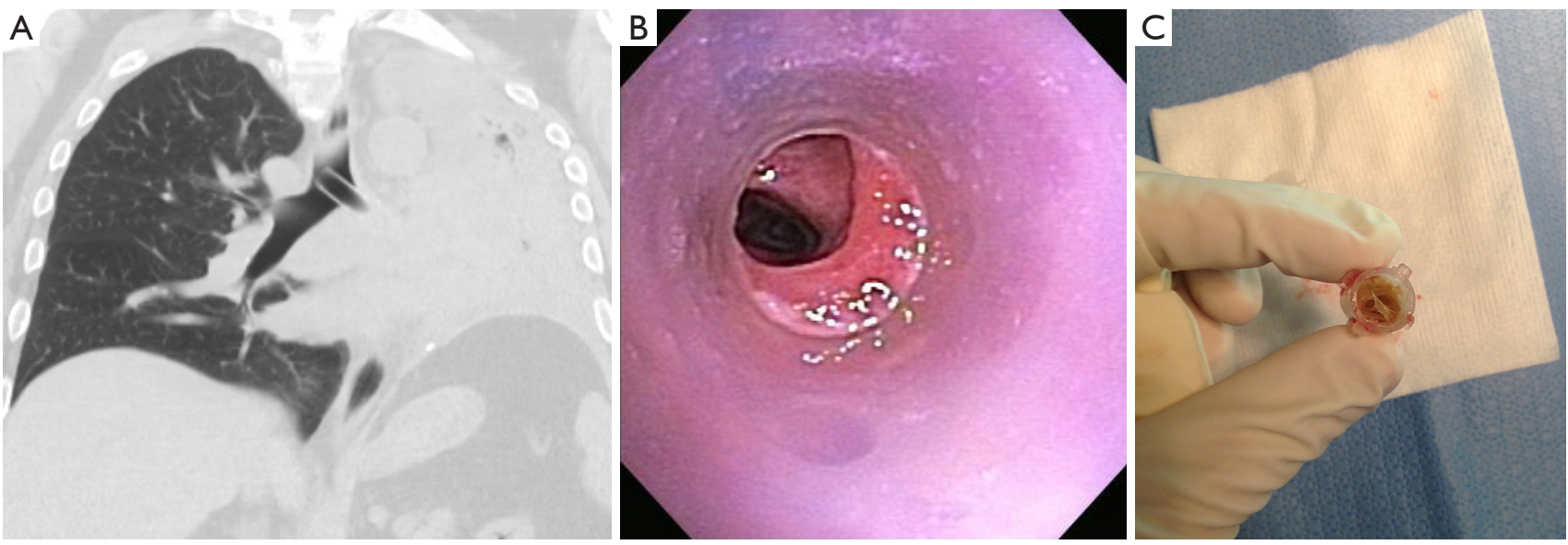

Figure 2 Common complications of silicone stents. (A) Silicone stent migration; (B) granulation at distal end of the stent; (C) mucus plugging in stent.
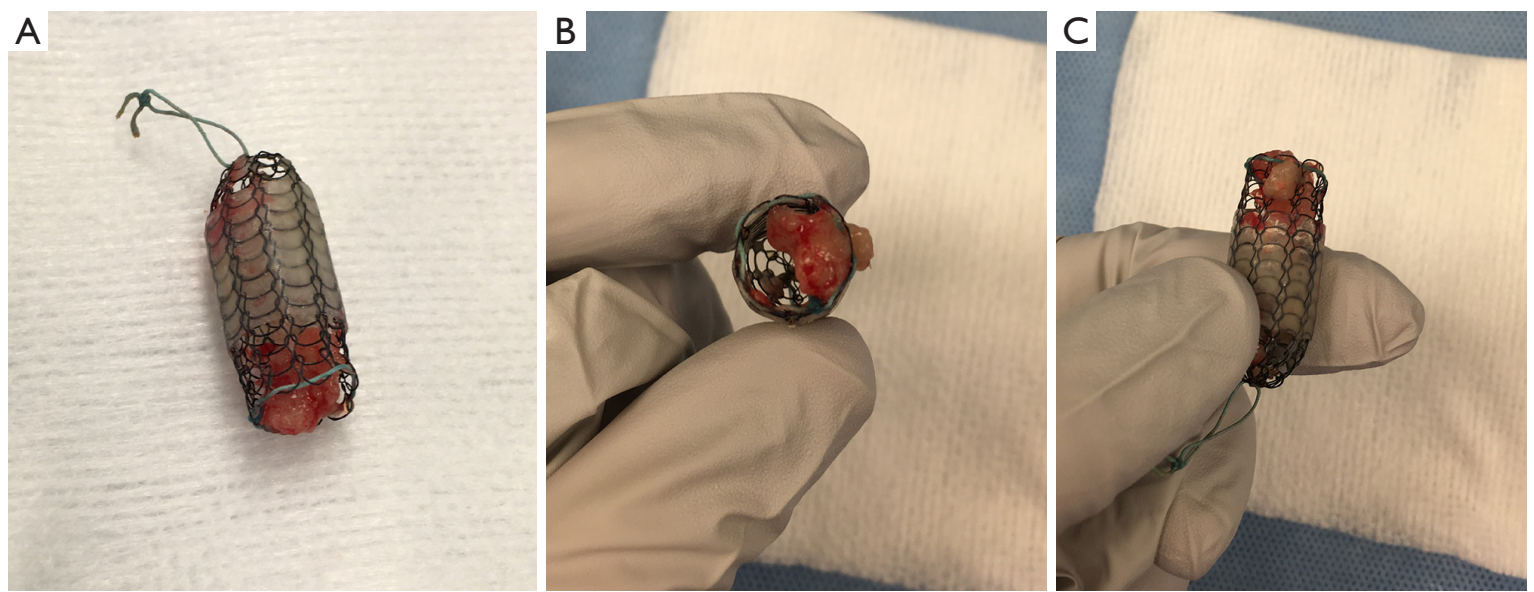

Figure 3 Ultraflex partially covered stent after successful removal. (A) Covered nitinol stent with proximal string for removal; (B) covered nitinol stent showing granulation tissue that penetrates the uncovered segment of the stent preventing migration but causing obstruction; (C) covered nitinol stent with granulation tissue that penetrates the uncovered segment. 


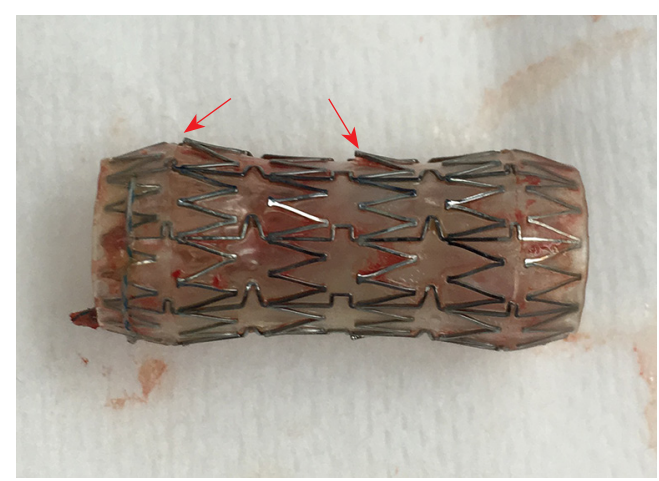

Figure 4 Merit Endotek nitinol stent with anti-migration fins (arrows) that may cause trauma during cough or attempted removal.

attachment was seen in this study. Stents have been designed with external or internal covering. The external cover does not allow the stents to incorporate into the tissue, leading to a migration rate estimated between $18 \%$ and $22 \%$ (25). Internally covered stents, on the other hand, are less susceptible to migration from friction and anchor of the bare metal outside of the stent in contact with the airway. A recent study showed a $4 \%$ migration rate with the use of an internal silicone-covered stent.

Some of the covered metallic stents have, at the proximal end, a small loop for manipulation that once grasped, will subsequently create a partial collapse of the stent to allow removal (Figure $3 A$ ). Among its disadvantages, the stent cover may facilitate sputum retention and obstruction, as is the case with silicone stents (13). Recently, self-expanding Y-stents became available outside of the United States. The initial experience in 38 patients with 3-month followup using rigid and flexible bronchoscopy placement is considered preliminary (26). Here, we present some of the most common metallic and hybrid stents used.

\section{Ultraflex stent (Boston Scientific, Natick, MA, USA)}

Ultraflex is a nitinol (nickel-titanium alloy) stent designed with a polyurethane cover to prevent tumor or granulation tissue ingrowth. It is deployed by a guide-wire technique or flexible bronchoscopy and is self-expandable (7). Some experts also deploy it through rigid bronchoscopy. The Ultraflex stent comes in a compressed state and is delivered to the desired area by gradually removing a suture that secures the stent in place. It has "shape memory"; it self-adjusts to changes in the bronchial size without any shortening after deployment, and it deforms at cold temperatures, while regaining its original shape at higher or body temperatures $(18,27)$.

\section{Polyflex stent (Boston Scientific, Natick, MA, USA)}

The Polyflex stent is a hybrid self-expanding stent made from a polyester-fiber wire mesh covered by silicone (28). It is deployed under rigid bronchoscopy using a special delivery device. It is available as a straight stent in various diameters and lengths. Migration rates have been described to be as high as $69 \%$, and a $25 \%$ rate of significant mucous retention has been reported (29). In a separate prospective study at 5-centers, researchers reported their experience with 27 Polyflex stents for malignant airway obstruction, where the migration rate was $3 \%$ (28).

\section{Merit Endotek or alveolus stent (Merit Medical Systems, South Fordan, Utab) (Figure 4)}

Alveolus is a fully covered hybrid stent with a dedicated deployment device and specially designed airway measurement device. It may be placed using fluoroscopic guidance, as well as rigid bronchoscopy. Limited data has been published on its use (30). The initial publications reported a significant risk of migration, prompting a design modification that included "non-migration" fins. These appendages become more prominent as pulling forces are exerted on the stent. As a result, the "anti-migration" fins sink deeper into the mucosa effectively preventing migration (see detail in Figure 4). Unfortunately, this may cause significant mucosal injury at the time of stent removal. In unique circumstances, our group has experience with customizing the length of this stent, by trimming rows of struts prior to deployment.

\section{The dynamic (Freitag) stent (Boston Scientific, Natick, $M A, U S A)$}

The Freitag "Y" silicone stent with bands of stainless steel (horseshoe-shaped) incorporated with silicone, along the anterior and lateral surface of the cylindrical tube to mimic tracheal cartilages. Thus, the posterior membrane is flexible and mimics the motion of the posterior tracheal wall during respiration (18,31). The rigidity of the dynamic $\mathrm{Y}$ stent precludes its use in conventional rigid stent deployers, and actually requires removal of the rigid bronchoscope for placement under direct laryngoscopy. It can also be deployed under suspension laryngoscopy. These stents are mostly useful in cases of tracheobronchomalacia and malignant airway disease involving the main carina. 


\begin{tabular}{|l|}
\hline Table 3 Complications of airway stents \\
\hline Stent migration \\
\hline Stent malposition \\
\hline Granulation formation \\
\hline Tumor ingrowth \\
\hline Mucoid impaction \\
\hline Infection \\
\hline Stent fracture \\
\hline Bronchovascular fistula \\
\hline Airway rupture \\
\hline Halitosis \\
\hline
\end{tabular}

\section{Stents for tracheoesophageal or bronchoesophageal fistulas}

Stents have been used to seal fistulas between the respiratory and digestive tracts when surgical correction is contraindicated. In cases where an esophageal stent has been placed, perforation and migration can be prevented by a tracheal stent. In fact, for the treatment of large tracheoesophageal fistulas, stenting of both the trachea and the esophagus seems to be superior over single stenting of either tract $(3,32,33)$. Collaboration with a surgeon is advised to determine the most appropriate remedy for this complicated condition.

\section{Airway stents in lung transplantation}

Bronchial anastomotic dehiscence after lung transplantation is a potentially catastrophic airway complication, seen typically within 1 to 5 weeks after the implantation. Fortunately, the incidence of severe dehiscence is low (23). The management depends on the symptoms and severity of the presentation. Therapy may involve observation for mild cases, versus surgical or endoscopic management for high-grade dehiscence. Mughal et al. described the successful management of severe dehiscence in seven patients using airway stents (21). In this series, Ultraflex uncovered stents were used as a platform for dehiscence healing. They took advantage of the uncovered metallic stents "complication" of excessive granulation tissue to heal the dehiscence.

\section{Comparative data in the lab}

A recent publication comparing three different tracheal stents made of steel, nitinol and nitinol drug-eluting compounds demonstrated the highest granuloma formation and overall proliferative reactivity with steel, followed by drug-eluting nitinol and finally nitinol being least reactive (34).

\section{Comparative data in clinical research}

To date, there are no comparative trials of stents in the bronchoscopy literature. Retrospective studies, with their shortcomings, have shown different risks of complications for different airway stents. In one such study, 172 patients with 195 stents showed Merit Endotek (former Aero) stents had an increased risk of infection (HR 1.98; 95\% CI, 1.03-3.81) and silicone tube stents had increased risk of migration (HR 3.52; 95\% CI, 1.41-8.82). Silicone stents and lower respiratory tract infections were associated with increased risk of granulation tissue (35).

\section{Complications of stents}

Airway stents have been associated with significant complications. The most important complications are associated with human error in placement, sizing and perhaps overextended placement. The initial stents and tubes (i.e., Montgomery T-tube) required a tracheostomy for placement. Thereafter, the rigid bronchoscope was used, and, in the last two decades, self-expanding metallic stents have been placed with rigid or flexible bronchoscopy. The complications associated with airway stents can derive from the following factors: the material; covering, or lack thereof; continuous movement; infection; mucociliary inhibition; or biomechanical properties of the stent. The original complications associated with metallic stents were the result of repurposing of colon, biliary or vascular stents for airway use. Their high radial force and traumatic borders were responsible for catastrophic complications and clinicians were forced to abandon their use or attempt sizing trickery to minimize migration and other complications. However, complications still occur. Table 3 describes common and uncommon complications of airway stents.

Some investigators consider that prompt removal of airway stents after remodeling of the airway has occurred, 
or after adequate response to chemotherapy or radiation, may minimize these complications. Furthermore, the use of scheduled follow-up with computed tomography or bronchoscopy to identify and address early complications is recommended (36).

\section{The case for lobar and segmental stents}

Traditionally, stent placement has been only recommended for central airways (trachea, mainstem bronchi) for two reasons: first, the available silicone stents had a $1 \mathrm{~mm}$ or $1.5 \mathrm{~mm}$ wall, conferring a disadvantage in the internal-toexternal diameter ratio, and; second, the idea that lobar or segmental salvage was not necessary given the limited survival of lung cancer. In the recent years, progress in lung cancer survival and technological improvements in the manufacturing process of airway stents has challenged these assumptions. A growing group of experts are using small metallic stents either in balloon expanding or self-expanding formats with encouraging results $(37,38)$.

\section{When the stent is temporary}

A rarely discussed situation in the airway stent literature is the use of temporary stents. In selected situations, the clinician may encounter a significant airway obstruction that requires stent placement with subsequent removal a few weeks later, example situations include:

* Temporary stent trial for evaluation of the potential clinical benefit of surgical tracheoplasty for tracheobronchomalacia. Our group and others have used short-term (1-2 weeks) trials with silicone Y-stents and uncovered nitinol stents in an attempt to identify those patients who are more likely to benefit from surgical tracheoplasty $(39,40)$. It is imperative to have a pre-procedural agreement with the patient that the stent(s) will only remain in place for 1-2 weeks given the potential complications of airway stents in benign disease. After completing subjective and objective evaluation of its benefit, the stent(s) are removed, where any low-grade granulation or irritation that may have occurred can be expected to resolve within days to weeks. Thereafter, those patients who responded may undergo surgical tracheoplasty;

* Airway stent(s) placed for tumors at the time of initial diagnostic bronchoscopy. In rare cases, the tumor causing the obstruction will have a dramatic initial response to chemotherapy or radiotherapy (e.g., lymphoma or small cell lung cancer). In these cases, and in absence of the final pathology, it is reasonable to place a palliative stent that may be removed at a later procedure;

* Airway stent(s) placed for airway dehiscence after lung transplantation. As previously described, these are rare situations where creating a scaffold for granulation and re-epithelization is warranted. After a few weeks, careful removal of the stent is carried out.

\section{How to remove stents}

The literature on stent removal is scant and mostly describes the complications related to inappropriate stent placement. The removal of silicone stents in general is straightforward. It involves rigid bronchoscopy and the use of rigid forceps to simultaneously twist-and-pull the stent into the barrel of the rigid bronchoscope while maintaining continuous visual contact with the stent. Twisting is thought to prevent tearing of the stent generated from single-plane pulling. In the case of Montgomery T-tubes, the removal just requires gentle yet firm tugging from the horizontal limb; caudally at first, to release the proximal limb, and then cephalad to remove the remaining distal limb.

The difficulty in removing metallic stents was initially described with the use of biliary stents adapted to the airway (41). Every effort should be made to remove the stent in one piece. This minimizes the risk of leaving stent fragments and also decreases the length of the procedure. We described our experience in removing metallic stents by using cryoablation, Jackson dilators or Fogarty balloons and rigid bronchoscopy (42). The removal of metallic stents in the first 2 months is relatively simple. However, after that interval, the degree of difficulty and experience necessary increases.

An important factor to consider is the specific type of metallic stent. Laser-cut fully covered stents (i.e., MeritEndotek) will have less tumor ingrowth, but the antimigration fins will likely cause significant mucosal injury and bleeding. After separating them from the wall with Jackson dilators or Fogarty balloon, these stents should be removed by rigid forceps in a twist-and-turn fashion similar to silicone stents. Braided nitinol stents (i.e., Ultraflex) should also be freed from the wall with Jackson or Fogarty balloons first. Thereafter, identify the proximal circumferential suture and pull from that point in order to purse-string the proximal end of the stent and facilitate removal (Figure 3). Failure to do so may result in piecemeal 


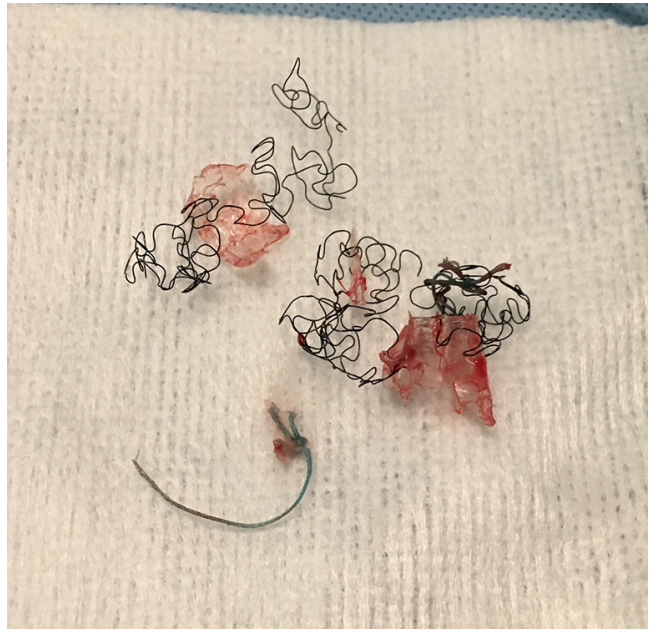

Figure 5 Poor removal technique of nitinol stent. Traction applied to the metal struts causes unraveling of the stent and complicates removal.

removal of the metallic wires (Figure 5). If flexible forceps are employed, a rat tooth grasping forceps is recommended, to reduce loss of the hold on the purse-string during removal. In all types of airway stents, it is important to try to remove the proximal and distal granulation tissue before attempting to remove them (42).

\section{The future of airway stents}

In the last 5 years, attempts to create bioabsorbable stents have been published $(43,44)$. These are still prototypes, and their long-term success and complication rates are uncertain. The prospect of minimizing long-term complications from airway stents is inspiring. Given the concern that stents are not always suitable for specific cases and that excessive radial forces exerted by the stent on the airway mucosa may be responsible for excessive granulation tissue, our group and others have explored the possibility of 3-D-printed airway stents $(8,45,46)$. This is a promising field as newer medical-grade materials become available.

\section{Acknowledgements}

None.

\section{Footnote}

Conflicts of Interest: Dr. Folch has served as a scientific consultant for Boston Scientific and Medtronic. Dr. Keyes has no conflicts of interest to declare.

\section{References}

1. Ernst A, Silvestri GA, Johnstone D, et al. Interventional pulmonary procedures: Guidelines from the American College of Chest Physicians. Chest 2003;123:1693-717.

2. Mathisen DJ, Morse CR. Thoracic surgery. Lung resections, bronchoplasty. Master techniques in surgery. Philadelphia: Wolters Kluwer, 2015.

3. Bolliger CT, Sutedja TG, Strausz J, et al. Therapeutic bronchoscopy with immediate effect: laser, electrocautery, argon plasma coagulation and stents. Eur Respir J 2006;27:1258-71.

4. Barros Casas D, Fernandez-Bussy S, Folch E, et al. Nonmalignant central airway obstruction. Arch Bronconeumol 2014;50:345-54.

5. FDA public health notification: Complications from metallic tracheal stents in patients with benign airway disorders. FDA. 2005. Available online: www.fda.gov/cdrh/ safety/072905-tracheal.html, accessed November 26, 2017.

6. Shea M, Huberman MS, Costa DB. LazarusType Response to Crizotinib in a Patient with Poor Performance Status and Advanced MET Exon 14 Skipping Mutation-Positive Lung Adenocarcinoma. J Thorac Oncol 2016;11:e81-2.

7. Wang KP, Mehta AC, Turner JF. Flexible bronchoscopy. 3rd ed. Chichester, West Sussex, UK: Wiley-Blackwell, 2012.

8. Cheng GZ, San Jose Estepar R, Folch E, et al. Threedimensional Printing and 3D Slicer: Powerful Tools in Understanding and Treating Structural Lung Disease. Chest 2016;149:1136-42.

9. Wood DE, Liu YH, Vallieres E, et al. Airway stenting for malignant and benign tracheobronchial stenosis. Ann Thorac Surg 2003;76:167-72; discussion 173-4.

10. Chen Q, Liang S, Thouas GA. Elastomeric biomaterials for tissue engineering. Progress in Polymer Science 2013;38:584-671.

11. Vearick SB, Demetrio KB, Xavier RG, et al. Fiberreinforced silicone for tracheobronchial stents: An experimental study. J Mech Behav Biomed Mater 2018;77:494-500.

12. Strausz J, Kis S, Pápai Z, et al. Tracheobronchial Silicone Stent Implantation with the Flexible Bronchoscope. J Bronchology Interv Pulmonol 1994;1:123-5.

13. Makris D, Marquette CH. Tracheobronchial stenting 
and central airway replacement. Curr Opin Pulm Med 2007;13:278-83.

14. Lemaire A, Burfeind WR, Toloza E, et al. Outcomes of tracheobronchial stents in patients with malignant airway disease. Ann Thorac Surg 2005;80:434-7; discussion 437-8.

15. Diaz-Jimenez JP, Muñoz EF, Martinez Ballarin JI, et al. Silicone Stents in the Management of Obstructive Tracheobronchial Lesions: 2-Year Experience. J Bronchology Interv Pulmonol 1994;1:15-8.

16. Dumon JF, Cavaliere S, Diaz-Jimenez JP, et al. Seven-Year Experience with the Dumon Prosthesis. J Bronchology Interv Pulmonol 1996;3:6-10.

17. Simoff MJ, Sterman DH, Ernst A. Thoracic endoscopy: advances in interventional pulmonology. Malden, Mass: Blackwell Futura, 2006.

18. Mehta AC, Dasgupta A. Airway stents. Clin Chest Med 1999;20:139-51.

19. Saad CP, Murthy S, Krizmanich G, et al. Self-expandable metallic airway stents and flexible bronchoscopy: longterm outcomes analysis. Chest 2003;124:1993-9.

20. Choudhary C, Bandyopadhyay D, Salman R, et al. Broncho-vascular fistulas from self-expanding metallic stents: A retrospective case review. Ann Thorac Med 2013;8:116-20.

21. Mughal MM, Gildea TR, Murthy S, et al. Short-term deployment of self-expanding metallic stents facilitates healing of bronchial dehiscence. Am J Respir Crit Care Med 2005;172:768-71.

22. Santacruz JF, Mehta AC. Airway complications and management after lung transplantation: ischemia, dehiscence, and stenosis. Proc Am Thorac Soc 2009;6:79-93.

23. Mahajan AK, Folch E, Khandhar SJ, et al. The Diagnosis and Management of Airway Complications Following Lung Transplantation. Chest 2017;152:627-38.

24. Hautmann H, Rieger J, Huber RM, et al. Elastic deformation properties of implanted endobronchial wire stents in benign and malignant bronchial disease: A radiographic in vivo evaluation. Cardiovasc Intervent Radiol 1999;22:103-8.

25. Kim JH, Shin JH, Song HY, et al. Use of a retrievable metallic stent internally coated with silicone to treat airway obstruction. J Vasc Interv Radiol 2008;19:1208-14.

26. Madan K, Dhooria S, Sehgal IS, et al. A Multicenter Experience With the Placement of Self-Expanding Metallic Tracheobronchial Y Stents. J Bronchology Interv Pulmonol 2016;23:29-38.
27. Vinograd I, Klin B, Brosh T, et al. A new intratracheal stent made from nitinol, an alloy with "shape memory effect". J Thorac Cardiovasc Surg 1994;107:1255-61.

28. Bolliger CT, Breitenbuecher A, Brutsche M, et al. Use of studded Polyflex stents in patients with neoplastic obstructions of the central airways. Respiration 2004;71:83-7.

29. Gildea TR, Murthy SC, Sahoo D, et al. Performance of a self-expanding silicone stent in palliation of benign airway conditions. Chest 2006;130:1419-23.

30. Mehta AC. AERO self-expanding hybrid stent for airway stenosis. Expert Rev Med Devices 2008;5:553-7.

31. Freitag L, Tekolf E, Stamatis G, et al. Clinical evaluation of a new bifurcated dynamic airway stent: a 5-year experience with 135 patients. Thorac Cardiovasc Surg 1997;45:6-12.

32. Freitag L, Tekolf E, Steveling H, et al. Management of malignant esophagotracheal fistulas with airway stenting and double stenting. Chest 1996;110:1155-60.

33. Rodriguez AN, Diaz-Jimenez JP. Malignant respiratorydigestive fistulas. Curr Opin Pulm Med 2010;16:329-33.

34. Serrano C, Lostale F, Rodriguez-Panadero F, et al. Tracheal Self-Expandable Metallic Stents: A Comparative Study of Three Different Stents in a Rabbit Model. Arch Bronconeumol 2016;52:123-30.

35. Ost DE, Shah AM, Lei X, et al. Respiratory infections increase the risk of granulation tissue formation following airway stenting in patients with malignant airway obstruction. Chest 2012;141:1473-81.

36. Dialani V, Ernst A, Sun M, et al. MDCT detection of airway stent complications: comparison with bronchoscopy. AJR Am J Roentgenol 2008;191:1576-80.

37. Sethi S, Wang J, Machuzak M, et al. Clinical Success Stenting Lobar and Segmental Bronchi for "Lobar Salvage" in Bronchial Stenosis. Chest 2014;146:732A.

38. Majid A, Kheir F, Chung J, et al. Covered BalloonExpanding Stents in Airway Stenosis. J Bronchology Interv Pulmonol 2017;24:174-7.

39. Majid A, Alape D, Kheir F, et al. Short-Term Use of uncovered self-expanding metallic airway stents for severe expiratory central airway collapse. Respiration 2016;92:389-96.

40. Ernst A, Majid A, Feller-Kopman D, et al. Airway stabilization with silicone stents for treating adult tracheobronchomalacia: a prospective observational study. Chest 2007;132:609-16.

41. Gaissert HA, Grillo HC, Wright CD, et al. Complication of benign tracheobronchial strictures by self-expanding 
metal stents. J Thorac Cardiovasc Surg 2003;126:744-7.

42. Majid A, Palkar A, Myers R, et al. Cryotechnology for staged removal of self-expandable metallic airway stents. Ann Thorac Surg 2013;96:336-8.

43. Dutau H, Musani AI, Laroumagne S, et al. Biodegradable Airway Stents - Bench to Bedside: A Comprehensive Review. Respiration 2015;90:512-21.

44. Freitag L, Gördes M, Zarogoulidis P, et al. Towards Individualized Tracheobronchial Stents: Technical, Practical and Legal Considerations. Respiration

Cite this article as: Folch E, Keyes C. Airway stents. Ann Cardiothorac Surg 2018;7(2):273-283. doi: 10.21037/ acs.2018.03.08
2017;94:442-56.

45. Cheng GZ, Folch E, Brik R, et al. Three-dimensional modeled $\mathrm{T}$-tube design and insertion in a patient with tracheal dehiscence. Chest 2015;148:e106-8.

46. Young BP, Machuzak MS, Gildea TR. Initial Clinical Experience Using 3D Printing and Patient-Specific Airway Stents: Compassionate Use of 3D Printed PatientSpecific Airway Stents. Am J Respir Crit Care Med 2017;195:A1711. 